anomalies, no increased risks in heart, limb or genital system were found.

Conclusion There are similar risks of major congenital anomalies in children born to women with and without IBD. No evidence of potential teratogenic effects of 5-aminosalicylic acid, steroids or azathioprine was found in this study. Previous guidance that women may be advised to continue these medications remains appropriate.

Disclosure of Interest None Declared

\section{OC-074 THE ROLE OF THE FUNGAL MICROBIOTA IN THE PATHOGENESIS OF DE-NOVO PAEDIATRIC INFLAMMATORY BOWEL DISEASE USING NEXT GENERATION SEQUENCING}

doi:10.1136/gutjnl-2013-304907.073

'R Hansen, 'I Mukhopadhya, ${ }^{2} \mathrm{C}$ Meharg, ${ }^{3} \mathrm{R}$ Russell, 'S Berry, 'E El-Omar, ${ }^{1, *} \mathrm{G}$ Hold. ${ }^{1}$ Division of Applied Medicine, Aberdeen University, Aberdeen; ${ }^{2}$ Queens University Belfast, Belfast; ' ${ }^{P}$ Paediatric Gastroenterology, Royal Hospital for Sick Children, Glasgow, UK

Introduction Paediatric Inflammatory bowel disease (IBD) incidence is rising worldwide. Recently the role of the gut microbiota has been recognised as pivotal in disease pathogenesis. IBD microbial studies to date have focused on bacterial diversity assessment in established disease cohorts, with limited studies in treatment naive patients. In contrast to bacteria, the exact role of the colonising fungi and their pathogenic potential has not been fully explored. The aim of the study was to examine candidate fungal triggers at disease onset in children with IBD using pyrosequencing, utilising the Bacteria in Inflammatory bowel disease in Scottish Children Undergoing Investigation before Treatment (BISCUIT) study cohort.

Methods 128 children undergoing colonoscopy were approached from three Scottish paediatric centres (Aberdeen, Glasgow and Dundee) with 100 ultimately recruited and biopsied; 44 IBD (comprising Crohn's disease (CD; 29), ulcerative colitis (UC; 13) and IBD-type unspecified (2)), 42 normal colon controls (NCC) and 14 "others". All IBD patient samples were taken from inflamed tissue. Fungal DNA was amplified on a reduced cohort of 37 recruits (13 CD, 12 UC, 12 NCC) using 18S rDNA primers. Roche 454 Titanium sequencing was conducted by NewGene (Newcastle, UK). Data analysis was performed using OIIME version 1.3.0 workflow. Taxonomy assignment of operational taxonomic units (OTUs) was performed according to ribosomal database project taxonomy. OTU tables were rarefied at 3,000 reads.

Results Fungal DNA was amplifiable from 7 patient samples, 6 children with a diagnosis of IBD - 4 with CD (BISCUIT1, 31, 62 and 89), 2 children with UC (BISCUIT33 and 104) and 1 NCC (BISCUIT 27). Fungal diversity was assessed in all paediatric samples alongside three adult samples to act as comparison. The adult samples comprised 1 patient with UC (2UC21Aa) and 2 NCC (GH4 and GH9). Phylum level analysis indicated that all fungal sequences belonged to the Ascomycota and Basidiomycota phyla. Control patients contained predominantly Ascomycota sequences ( $>80 \%$ of sequences in all patients) whilst $6 / 7$ IBD patients contained exclusively Basidiomycota species. Genus level analysis was undertaken and there was no similarity between fungal profiles from the paediatric and adult samples.

Conclusion By using robust methodology we have characterised the IBD "fungal microbiota" at diagnosis in children. Based on the current study, it would appear that a distinctly altered fungal species profile is present at IBD disease presentation. Further work should now focus on expanding this study and identifying how to beneficially modify the microbiota using established and novel IBD treatments.

Disclosure of Interest None Declared

\section{Endoscopy symposium: how I do it - ERCP}

\section{OC-075 ANALYSIS OF LONG-TERM OUTCOMES AFTER ENDOSCOPIC RADIOFREOUENCY ABLATION FOR BILE DUCT STRICTURES IN PANCREATIC MALIGNANCY SUGGESTS POTENTIAL SURVIVAL BENEFIT}

doi:10.1136/gutjnl-2013-304907.074

$1,2,{ }^{*} \mathrm{Y}$ Kallis, ${ }^{2} \mathrm{~N}$ Phillips, ${ }^{3} \mathrm{~A}$ Steel, ${ }^{2 \mathrm{H}}$ Kaltsidis, ${ }^{2} \mathrm{~J}$ Nicholls, ${ }^{2} \mathrm{~L}$ Jiao, ${ }^{2} \mathrm{P}$ Vlavianos, ${ }^{2} \mathrm{~N}$ Habib, ${ }^{2} \mathrm{D}$ Westaby. 'Endoscopy, Royal London Hospital, Barts Health NHS Trust; ${ }^{2} H P B$ Unit, Imperial College Healthcare NHS Trust; ${ }^{3}$ Gastroenterology, Chelsea \& Westminster NHS Foundation Trust, London, UK

Introduction Pancreatic carcinoma carries a poor prognosis with only $10-20 \%$ of patients amenable to attempts at curative surgery at presentation. Biliary obstruction is a common complication and many patients will require self-expanding metal stent (SEMS) insertion for definitive decompression. A recent pioneering phase I/II study in our tertiary referral centre demonstrated excellent safety and $90 \mathrm{~d}$ stent patency with endobiliary radiofrequency ablation (RFA) as an adjunct to SEMS insertion. The longer-term impact of this novel endoscopic treatment modality on biliary drainage and patient survival in advanced pancreatic carcinoma is unknown.

Objective To investigate the longer-term efficacy of endobiliary RFA in the management of malignant bile duct obstruction associated with inoperable pancreatic carcinoma.

Methods Retrospective cohort analysis of 23 patients with unresectable pancreatic carcinoma undergoing RFA + SEMS insertion, and 46 matched controls undergoing SEMS insertion alone, for malignant biliary obstruction in a single tertiary referral centre. Patients were stringently matched for age, sex, metastases, ASA/ co-morbidities, and intention to treat with palliative chemotherapy. Survival, maintenance of stent patency, and procedure-related complications were assessed.

Results RFA and control groups were closely matched- age 68.9 $+/-9.0 y$ vs. $69.8+/-9.9 y, p=0.791$; ASA $2.35+/-0.65$ vs. $2.54+/-$ $0.50, p=0.086$; metastases at treatment $9 / 23(39.1 \%)$ vs. $18 / 46$ (39.1\%), p = 0.800; chemotherapy $16 / 23(69.6 \%)$ vs. $24 / 46(52.2 \%)$, $p=0.203$. Median survival was $227 d$ after RFA vs. $123.5 \mathrm{~d}$ in controls (HR 0.633 CI 0.378-1.060, $\mathrm{p}=0.011$ ). RFA was independently predictive of survival at 90d (OR 16.14, CI 1.35-193.18, $p=0.028)$ and 180d (OR 4.25, CI 1.00-18.01, $\mathrm{p}=0.049$ ). Overall SEMS patency rates were the same across both groups, though more patients were alive with a patent index SEMS after RFA within the first few months $(73.9 \%$ vs. $41.3 \%$ at $4.5 \mathrm{~m}, \mathrm{p}=0.012)$. Complications of RFA were few (1 pancreatitis, 1 cholangitis), with a median post-procedure inpatient stay of $1 \mathrm{~d}(1-8)$.

Conclusion In the single largest case series to date, endobiliary RFA was found to be a safe and efficacious adjunctive treatment in the management of patients with advanced pancreatic malignancy and biliary obstruction, and demonstrated potential early survival benefit. These data suggest that endobiliary RFA could be an additional treatment option in advanced pancreatic carcinoma, and form the basis from which future prospective clinical trials of this novel treatment modality can be designed.

Disclosure of Interest Y. Kallis: None Declared, N. Phillips: None Declared, A. Steel: None Declared, H. Kaltsidis: None Declared, J. Nicholls Shareholder of: EMcision Ltd UK, L. Jiao: None Declared, P. Vlavianos: None Declared, N. Habib Shareholder of: EMcision Ltd UK, D. Westaby: None Declared.

\section{OC-076 SYRINGE SIZE INFLUENCES THE AMOUNT OF MIDAZOLAM ADMINISTERED DURING SEDATED ENDOSCOPY}

doi:10.1136/gutjnl-2013-304907.075

1." D Haldar, 'N Quraishi, 'K Glover, 'S Keen, 'A D Farmer. 'Gastroenterology, Shrewsbury \& Telford NHS Trust, Telford, UK 
Abstract 0C-076 Table 1

\begin{tabular}{llll}
\hline & Period 1 (x1 5ml midazolam syringe) & Period 2 (x2 2.5ml midazolam syringe) & P value \\
\hline Male:Female & $4770: 4990$ & $4910: 4948$ & 0.15 \\
Age +/- standard error of the mean (SEM) & 63.1 yrs $+/-0.6$ & $63.2+/-0.4$ & 0.88 \\
Total number of endoscopic procedures & 9760 & 9828 & 0.63 \\
Sedation proportion & $65.2 \%$ & $66 \%$ & $<0.0001$ \\
Midazolam dosage (+/- SEM) & $2.97+/-0.05$ & $2.51+/-0.02$ & 0.45 \\
Comfort scores & $1.1+/-0.008$ & $1.0+/-0.004$ & 0.98 \\
Complications & $0.08 \%$ & $0.07 \%$ & \\
\hline
\end{tabular}

Introduction Endoscopy remains important diagnostic tools for evaluating disease, early detection of malignant lesions and the delivery of therapy within the gastrointestinal (GI) tract. There are a number of regimens that are utilised for the administration of intra-venous sedation, including benzodiazepines (BDZ). However, sedation for endoscopy is not without its risks with an influential report suggesting that many major complications arise from patients being over-sedated (1). Thus, factors that are utilised to reduce the amount of sedation, without a detrimental effect on intra-procedural comfort, are important. Thus the aim of our study was to assess the impact of a change in syringe size of BDZ offered to endoscopists on the amount of BDZ administered in sedated GI endoscopy.

Methods A 2 centre, prospective trial comparing BDZ use across all forms of sedated endoscopy across two time periods. In the first period ( $1^{\text {st }}$ November 2010- 31 $1^{\text {st }}$ October 2011) endoscopists were given a single $5 \mathrm{ml}$ syringe containing $5 \mathrm{mg}$ midazolam. In the second time period ( $1^{\text {st }}$ November $2011-31^{\text {st }}$ October 2012) endoscopists were given two $2.5 \mathrm{ml}$ syringes each containing $2.5 \mathrm{mg}$ midazolam. BDZ use, comfort scores and complications were compared across the two time periods. Patients who had a general anaesthetic, and those who did not have sedation, of their endoscopy were excluded.

Results Table 1 details the results. Significantly less BDZ was administered in the second time period without an impact on comfort scores. Complications related to sedation were not different.

Conclusion Offering endoscopists smaller syringes reduces $\mathrm{BDZ}$ use but not at the expense of comfort levels. Further longitudinal study is required to further evaluate whether reduced BDZ usage has an impact on complications.

Disclosure of Interest None Declared

\section{REFERENCE}

1. Scoping Our Practice, The 2004 National Confidential Enquiry into Patient Outcomes and Death (NCEPOD), London, UK.

\section{BSG nurses}

\section{OC-077 IS THE REFERRAL APPROPRIATE?}

doi:10.1136/gutjnl-2013-304907.076

1."J Jones, "I Dunkley, 'J Maddocks. 'Hinchingbrooke Healthcare NHS Trust, Huntingdon, UK

Introduction With issues of increasing demand for endoscopy procedures, there is a need to validate surveillance referrals. A nurse-led review of surveillance referrals was set up. The aim was to ensure that patients are seen at the right time based on current guidance right patient, right time, right test.

Methods A protocol was developed which outlined the process for nurses to review referrals for patients on planned surveillance waiting list for colonoscopy. All referrals were reviewed with the hospital notes; relevant information documented on the protocol form which included changes in health; medication etc. Referrals with no change followed the pathway of original planned care. Referrals which did not meet the guidance (coded by nurses as: no/unsure) were reviewed by consultant colorectal surgeons. After consultant review if there were changes to planned care both the patient and their GP's were informed by letter of the changes.

Results A total of 296 surveillance referrals were reviewed between March - November 2012. Of these referrals were 126 were appropriate 170 were identified as needing changes to planned care. Of the 170 patients where changes were recommended, 115 were removed from the waiting list. 22 patients had a date change and 33 patients had no change to their planned care. Issues found for patients were: age, mental capacity, patient choice consideration, and documentation were: old versions or incomplete/illegible referrals, initial consultation documentation did not always include family history. See diagrams below: Conclusion Nurses are able to identify changes to planned surveillance colonoscopy referrals. Reducing risk to patients through inappropriate colonoscopy procedures; reducing waiting list and releasing capacity to other patients. This review shows there is a

\section{Outcome Following Consultant Review}

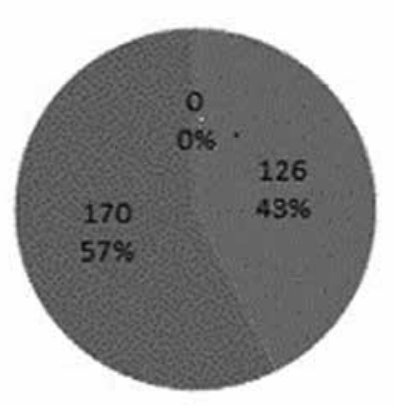

ayes

Eo/Unsure

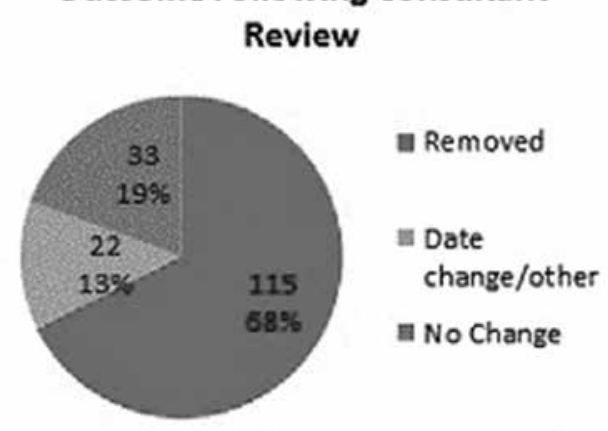

